\title{
Thermal Analysis of GaN/SiC-on-Si Assemblies: Effect of Bump Pitch and Thickness of SiC Layer
}

\author{
Kimmo Rasilainen ${ }^{* 1,3}$, Torbjörn M. J. Nilsson ${ }^{1,2}$, Johan Bremer ${ }^{1}$, Mattias Thorsel1 ${ }^{1,2}$, and Christian Fager ${ }^{1}$ \\ ${ }^{1}$ Department of Microtechnology and Nanoscience, Chalmers University of Technology, Gothenburg, Sweden \\ ${ }^{2}$ Saab AB, Gothenburg, Sweden \\ ${ }^{3}$ Centre for Wireless Communications, University of Oulu, Oulu, Finland \\ * Corresponding Author: kimmo.rasilainen@oulu.fi
}

\begin{abstract}
The ever-increasing requirements for high device performance and compact size drive the communications industry to look for new materials, technologies, and integration concepts. This simulation-based study investigates the thermal properties of a compact, heterogeneously integrated gallium nitride on silicon carbide (GaN-on-SiC) and silicon (Si) assembly. Thermal simulations and parametric studies are used to determine how the heat spreading and temperature levels in the lateral and vertical directions are affected by the thickness of the SiC layer and the distribution of the thermal interconnects. Results show that a SiC layer thinned down to $100 \mu \mathrm{m}$ shows more pronounced differences in its thermal characteristics compared to thicker ones, especially in terms of its backside heating. Aspects related to practical implementations are also considered.
\end{abstract}

\section{Introduction}

Current and future communications systems should provide more advanced functionality, improved energy efficiency, and smaller devices. As an example, the upcoming fifth generation (5G) communications systems [1], [2] require better spatial control of the radiated power by means of, e.g., beam steering and other sophisticated techniques. This increases the requirements for the electronics and typically also the power consumption. Combining high power levels and compact designs is not a trivial task, and this has become a hot research topic in the electronics packaging community.

An approach that has become increasingly popular is heterogeneous integration, which combines semiconductor technologies with complementary properties to improve overall performance [3], [4]. An attractive combination is gallium nitride $(\mathrm{GaN})$ that supports high power levels and silicon $(\mathrm{Si})$ that enables high integration density and technical maturity. To allow for more heat transfer from the GaN devices, they are typically grown on a silicon carbide $(\mathrm{SiC})$ carrier with high thermal conductivity.

As a technology, heterogeneous integration can be applied to various radio frequency $(\mathrm{RF})$, integrated circuit (IC) and System-in-Package (SiP) applications [5]-[7]. For proper functionality and reliability, it is important to understand the multi-physical (e.g. electrothermal) properties of the integrated systems to avoid design bottlenecks [8]-[10].

Figure 1 presents a conceptual drawing of a building practice suitable for compact millimetre-wave communications and sensing systems operating at, e.g., 20-50 GHz. In this concept, which utilises the heterogeneous integration of $\mathrm{GaN}$ and $\mathrm{Si}$, the low-power and high-density baseband/lowfrequency components can be manufactured in a Si process, and power amplifiers (PAs) and other high-power devices can be implemented on the $\mathrm{GaN} / \mathrm{SiC}$ layer. In a practical application, the Si chip would be fed from the backside with multiple inputs/outputs (I/Os), and a smaller number of I/Os is applied to the $\mathrm{GaN} / \mathrm{SiC}$ chip. This kind of stacked structure (where an antenna layer is located above the $\mathrm{GaN} / \mathrm{SiC}$ chip, see Figure 1) helps to reduce both the number of signal interconnects and the overall losses between the different chips. Such a vertical assembly also enables a compact footprint for the entire system.

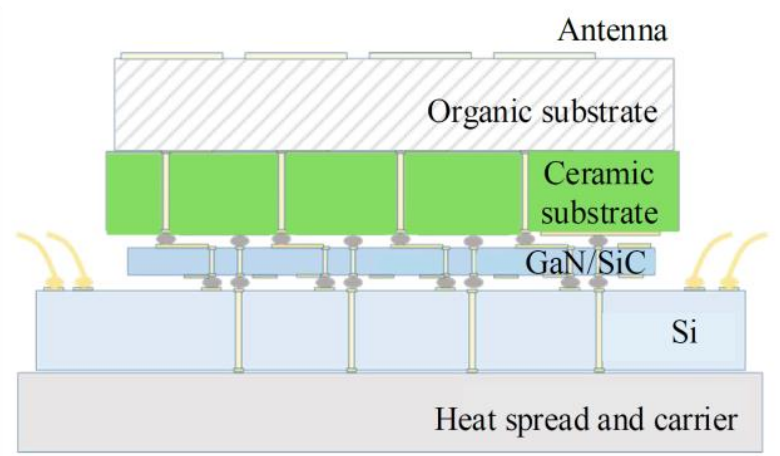

Figure 1. Conceptual drawing of a building practice for wireless communications and sensing applications at $\mathrm{mm}$ wave frequencies. The drawing is not entirely to scale.

This simulation-based study looks at differences in the heat spreading and thermal characteristics of a stacked $\mathrm{GaN} / \mathrm{SiC}$ on-Si assembly when the thickness of the $\mathrm{SiC}$ carrier and pitch of the thermal interconnects are changed. Numerical and experimental studies on thermal effects in flip-chip based assemblies and $\mathrm{GaN}$ devices have been reported in [11] and [12], respectively. For IC applications, effects of the thickness and distribution of through-silicon vias (TSVs) have been investigated in, e.g., [13], [14]. Recently, the thermal properties of different thermal interconnect types 
were studied in [15] for a more complicated geometry, but the effect of $\mathrm{SiC}$ carrier thickness was not considered.

\section{Investigated Stacked Assembly and Research Methodology}

The basic design investigated in this work consists of a $\mathrm{GaN} / \mathrm{SiC}$ chip mounted onto a Si chip using gold ( $\mathrm{Au}$ ) bumps. Thermal simulations are carried out using the ANSYS Icepak software (v. 19.1), and the simulations assume the material properties shown in Table 1.

Table 1: Properties of the materials used in the study.

\begin{tabular}{|c|c|c|c|}
\hline Material & $\begin{array}{c}\text { Density } \\
\rho\left(\mathrm{kg} / \mathrm{m}^{3}\right)\end{array}$ & $\begin{array}{c}\text { Thermal cond. } \\
k(\mathrm{~W} / \mathrm{m} \cdot \mathrm{K})\end{array}$ & $\begin{array}{c}\text { Specific heat } \\
C_{p}(\mathrm{~J} / \mathrm{kg} \cdot \mathrm{K})\end{array}$ \\
\hline $\mathbf{A u}$ & 1930 & 310 & 125 \\
\hline $\mathbf{S i}$ & 2330 & 180 & 770 \\
\hline $\mathbf{S i C}$ & 3210 & 450 & 1200 \\
\hline $\mathbf{G a N}$ & 6500 & 130 & 490 \\
\hline
\end{tabular}

\subsection{Chip Dimensions and Heat Sources}

Figure 2 illustrates the principal geometry of the study. The area of the Si chip is assumed to be $7 \times 7 \mathrm{~mm}^{2}$, and the size of the $\mathrm{GaN} / \mathrm{SiC}$ chip is $3 \times 3 \mathrm{~mm}^{2}$ (see Figure 3 ). A constant thickness of $400 \mu \mathrm{m}$ is used for the Si substrate. On top of the $\mathrm{SiC}$ carrier is a layer of $\mathrm{GaN}$ whose thickness is $3 \mu \mathrm{m}$. The thermal boundary resistance (TBR) in the $\mathrm{GaN} / \mathrm{SiC}$ interface is assumed to be $3.3 \cdot 10^{-8} \mathrm{~m}^{2} \cdot \mathrm{K} / \mathrm{W}$. The TBR is caused by a thin aluminium nitride (AIN) layer needed to grow the $\mathrm{GaN}$ layer on the $\mathrm{SiC}$ carrier. In a $\mathrm{GaN} / \mathrm{SiC}$ structure, the bulk $\mathrm{SiC}$ material acts as the main thermal carrier [16].

For the $\mathrm{SiC}$ carrier, its thickness is varied from 100 to $500 \mu \mathrm{m}$ with $100-\mu \mathrm{m}$ steps. The thickest substrate represents a standard thickness for typical commercially available $4 \mathrm{H}-\mathrm{SiC}$ wafers. In many practical applications, thinned-down substrates are desirable, which motivates the choice of the thinnest case. To complement the analysis, and to see more of how the heat spreading depends on the thickness, additional intermediate thickness values $(200,300$, and $400 \mu \mathrm{m})$ are investigated. It should be noted that most of the reported results are for the 100,300 , and $500-\mu \mathrm{m}$ cases.

In the interface between the two substrates, the bottom face of the $\mathrm{GaN} / \mathrm{SiC}$ chip and the top face of the Si chip are metallised with a thin Au layer, the thickness of which is 2 $\mu \mathrm{m}$. Considering practical assembly and manufacturing, such a thickness is suitable to connect the $\mathrm{GaN} / \mathrm{SiC}$ and $\mathrm{Si}$ chips using, e.g., thermocompression bonding and stud bumps.

To represent the high-power GaN device, a $10 \times 100 \mu \mathrm{m}^{2}$ heat source is located at the centre of the $\mathrm{GaN} / \mathrm{SiC}$ chip. The source dissipates a power of $5 \mathrm{~W}$, and the resulting power density causes a very concentrated local hotspot and impractically high temperatures exactly at the source. Outside of the source and in the different layers, the temperature remains at a technologically tolerable level. To represent the connection of the assembly to its thermal surroundings, a fixed heat transfer coefficient of $6000 \mathrm{~W} / \mathrm{m}^{2} \cdot \mathrm{K}$ is applied to the bottom interface of the Si substrate. This approach gives more accurate results than assuming a fixed temperature [17].

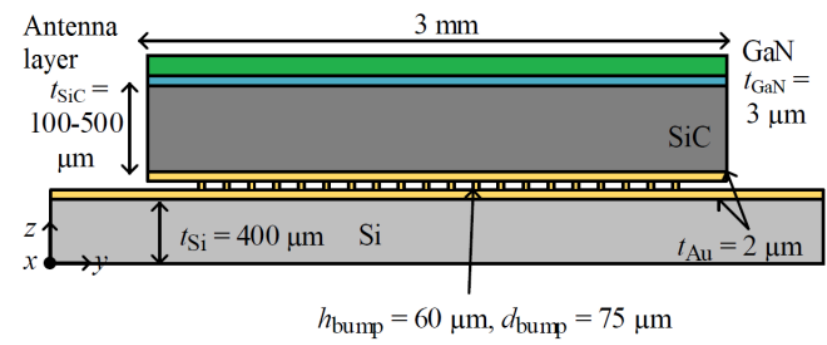

Figure 2. Side view of the investigated stacked assembly. The drawing is not entirely to scale, and the properties of the antenna layer are not considered in this study.

\subsection{Distribution of Au Bump Interconnects}

Figure 3 presents four different variants of Au bump arrays used to connect the two chips together. The height and diameter of the bumps are set to 60 and $75 \mu \mathrm{m}$, respectively, and these values are kept constant throughout the study. These bump dimensions showed good thermal properties in an earlier study [15], and they can also be considered feasible from a practical fabrication point of view. In all cases, the bumps are organised in a regular and symmetric array.

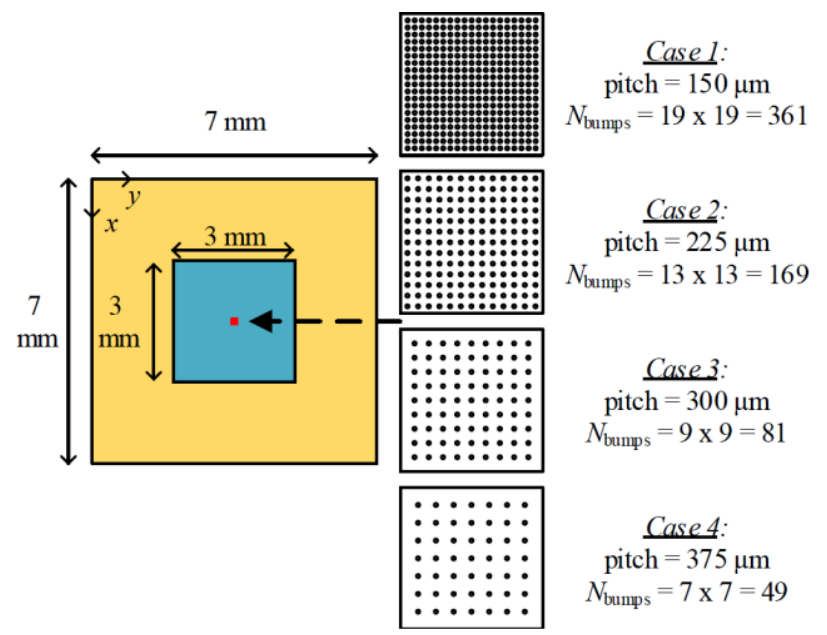

Figure 3. Top view of the assembly and the distribution of the Au bumps at the interface between the GaN/SiC and Si chips. The bump array is fully symmetric. Red square represents the heat source (not to scale).

The configuration of the Au bumps is varied by modifying their pitch as integer multiples of the bump diameter, and the pitch values used are $150,225,300$, and $375 \mu \mathrm{m}$. With these values, the arrays have $19 \times 19=361,13 \times 13=169,9 \times 9=81$, and $7 \times 7=49$ bumps, respectively, between the GaN/SiC and $\mathrm{Si}$ chips. In all cases, the bump array is symmetric with respect to the centre of the chip, and the centremost bump is always located directly below the heat source. 


\section{Simulated Thermal Characteristics}

For analysing the thermal properties of the investigated assembly, the lateral and vertical thermal characteristics of the structure are studied. This is done by observing temperature profiles and differences at various locations in the assembly. Geometric symmetry in the structure helps to reduce the number of cases to be considered. All dimensions and properties are kept constant throughout the study except for the bump pitch and $\mathrm{SiC}$ carrier thickness, which means that the observed thermal effects are mainly caused by these parameters.

\subsection{Lateral Thermal Characteristics}

The first step in the analysis is to investigate the heat spreading from the source in the lateral direction. Structural symmetry, in terms of the location of the heat source and bumps, allows limiting this study to one lateral path (parallel to the edges of the chips). Figure 4 presents the temperature profile at different layers of the assembly with different substrate thicknesses. These profiles are along a direction that crosses the centre of the $\mathrm{GaN} / \mathrm{SiC}$ chip and the heat source.

As the substrate gets thinner, the temperature at the bottom layer of the $\mathrm{SiC}$ carrier increases in the vicinity of the heat source, and a periodic pattern caused by the bump pitch can be seen in the temperature profile. With the thinnest substrate, the difference in the $\mathrm{SiC}$ bottom temperature is approximately $12{ }^{\circ} \mathrm{C}$ between the sparsest (pitch $=375 \mu \mathrm{m}$ ) and densest (pitch $=150 \mu \mathrm{m}$ ) bump grids. The symmetry of the bump array is evident from the curves of Figure 4. Having the centremost bump beneath the heat source causes the local minima seen at zero location in all the cases in Figure 4.

Figure 5 compares the temperature behaviour in the top surface of the GaN layer and the bottom surface of the $\mathrm{SiC}$ carrier as a function of the bump pitch. As the curves of Figure 5 show, the two thickest substrates have rather similar temperature characteristics. The temperature falls quite rapidly when moving away from the centre, and the largest temperature drop at the top face occurs within $250 \mu \mathrm{m}$ from the centre. With all substrates, the temperature profile of the bottom face begins to show periodic local minima corresponding to the pitch between adjacent bumps. This effect is most pronounced when the pitch is 300 and $375 \mu \mathrm{m}$.

When the SiC carrier is thinned down to $100 \mu \mathrm{m}$, the thermal behaviour in the vicinity of the source is different. Temperature drops comparable to those with the thicker chips (e.g. top side temperature) take place across a longer distance, and beyond approximately $100 \mu \mathrm{m}$ from the source, both sides of the $\mathrm{GaN} / \mathrm{SiC}$ chip have very similar temperature profiles. Adjacent to the source, the bottom face of the chip experiences more significant heating compared to the thicker substrates. The resulting temperature increase is up to $20^{\circ} \mathrm{C}$ both locally (bottom surface of the $\mathrm{SiC}$ carrier) and globally (GaN layer; see the curves of Figure 7).

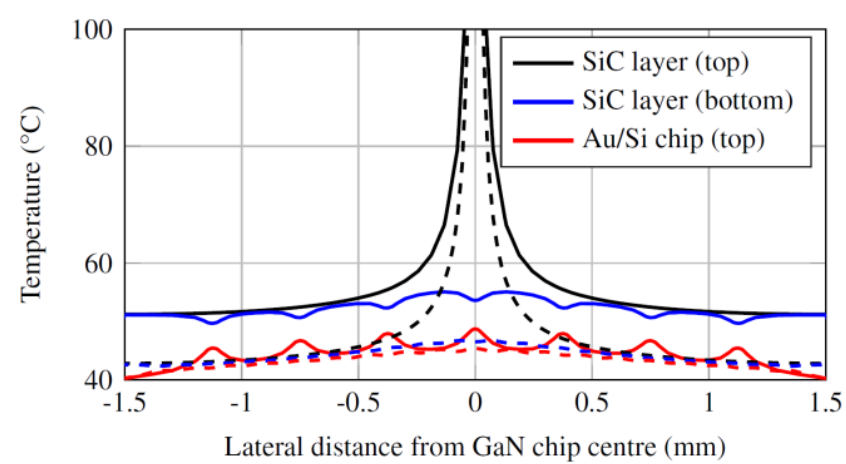

(a)

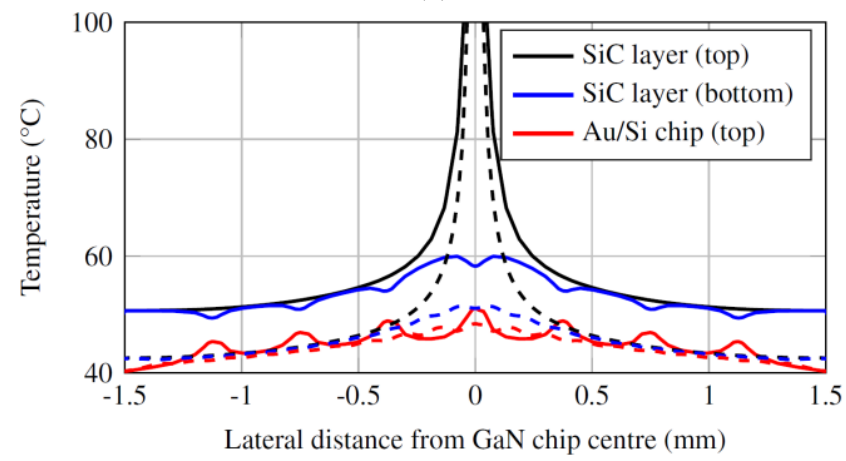

(b)

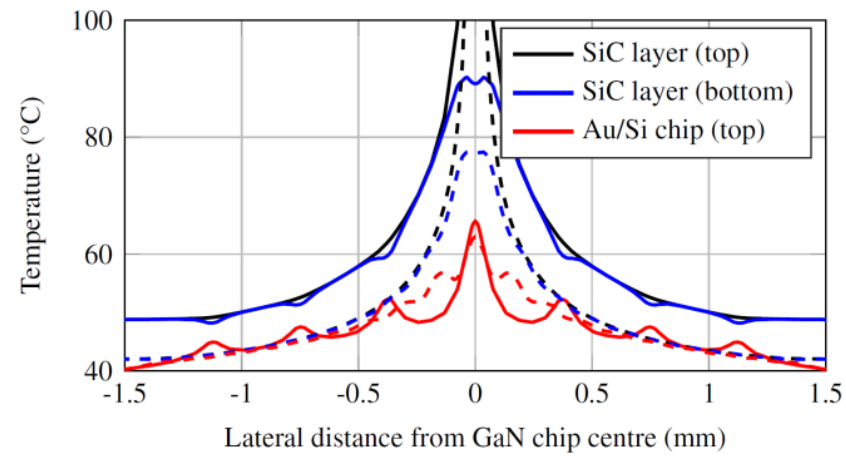

(c)

Figure 4. Comparison of temperature profiles at different layers in the assembly when the SiC carrier thickness is (a) $500 \mu \mathrm{m}$, (b) $300 \mu \mathrm{m}$, and (c) $100 \mu \mathrm{m}$. Bump pitch is $375 \mu \mathrm{m}$ (solid line) and $150 \mu \mathrm{m}$ (dashed line).

\subsection{Vertical Thermal Characteristics}

Figure 6 shows the vertical temperature profile through the centre of the $\mathrm{GaN} / \mathrm{SiC}$ chip (temperature drop from the top surface of the $\mathrm{GaN}$ layer to the bottom surface of the $\mathrm{SiC}$ carrier). The curves of Figure 6 illustrate that in the vertical direction, the temperature drop between the top and bottom surfaces of the chip is steeper with a thicker carrier. For example, the temperature at the bottom face of the $500-\mu \mathrm{m}$ carrier is approximately $20^{\circ} \mathrm{C}$ lower than in the $100-\mu \mathrm{m}$ case in the vicinity of the heat source.

Figure 7 presents a close-up of the thermal characteristics in the immediate vicinity of the top layer and $\mathrm{GaN} / \mathrm{SiC}$ interface within the first $20 \mu \mathrm{m}$ below the surface. As an example, the case of a $500-\mu \mathrm{m}$ thick $\mathrm{SiC}$ carrier is shown in Figure 7(a). Additionally, the thickness dependence is illustrated for the 
$150-\mu \mathrm{m}$ and $375-\mu \mathrm{m}$ bump pitch in Figures 7(b) and (c), respectively. Apart from slight variations in the absolute temperature levels, the general trend is similar also with thinner carriers. When the $\mathrm{SiC}$ carrier is $100 \mu \mathrm{m}$ thick, the shape of the $T_{\text {vert }}$ curves is different, and it displays up to three different slopes across the thickness.

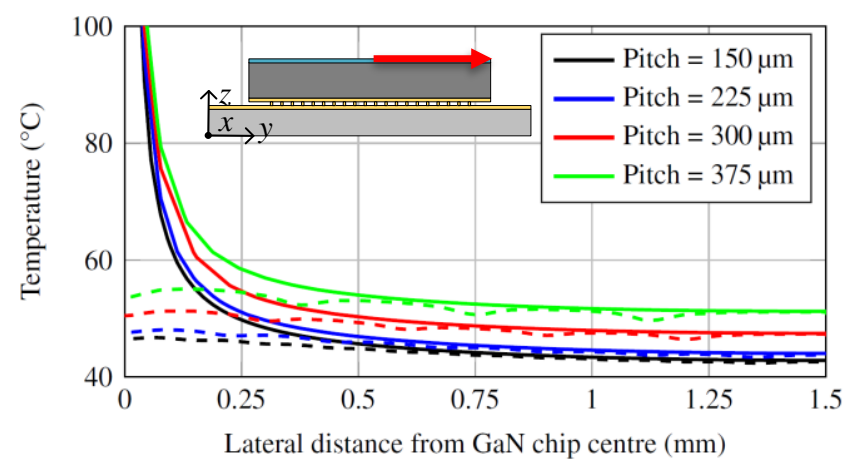

(a)

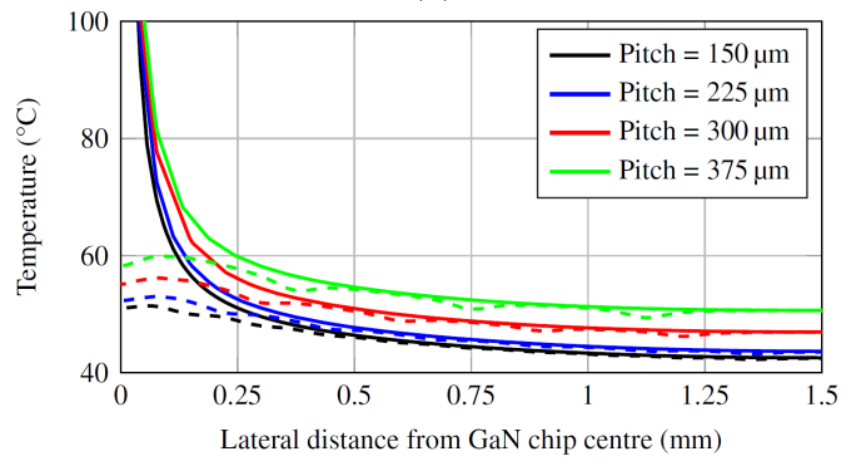

(b)

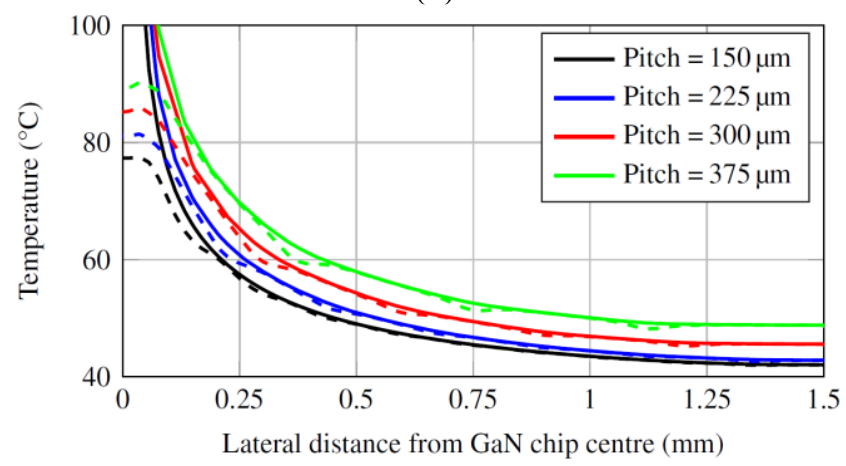

(c)

Figure 5. Lateral temperature profiles with different bump pitch values when the SiC carrier thickness is (a) $500 \mu \mathrm{m}$, (b) $300 \mu \mathrm{m}$, and (c) $100 \mu \mathrm{m}$. Solid line: top surface of the GaN layer. Dashed line: bottom surface of the SiC layer. The red line in the inset shows the direction of the temperature profile.

At the geometric centre of the source, the local peak temperature exceeds $400{ }^{\circ} \mathrm{C}$ (with the currently assumed value of $P_{\text {diss }}=5 \mathrm{~W}$ ), but the temperature gradient is extremely steep when moving away from this point. The GaN layer is $3 \mu \mathrm{m}$ thick, and the thermal boundary resistance at the interface affects the thermal properties (seen as a large discontinuity or change of slope in the temperature profile).

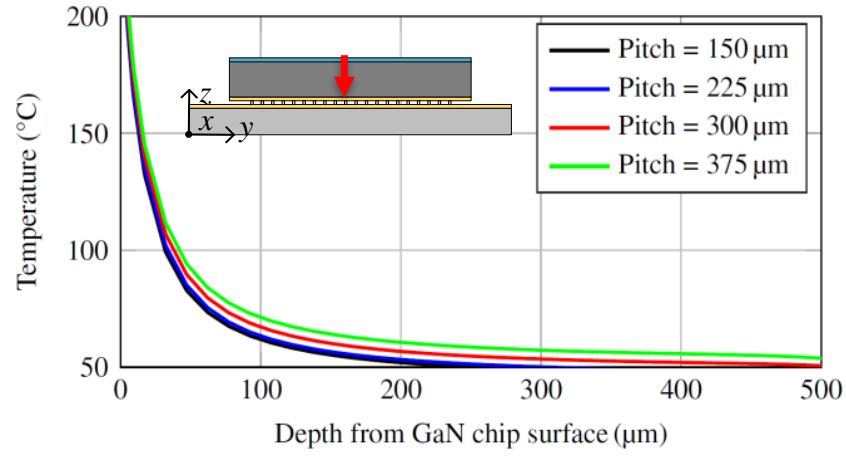

(a)

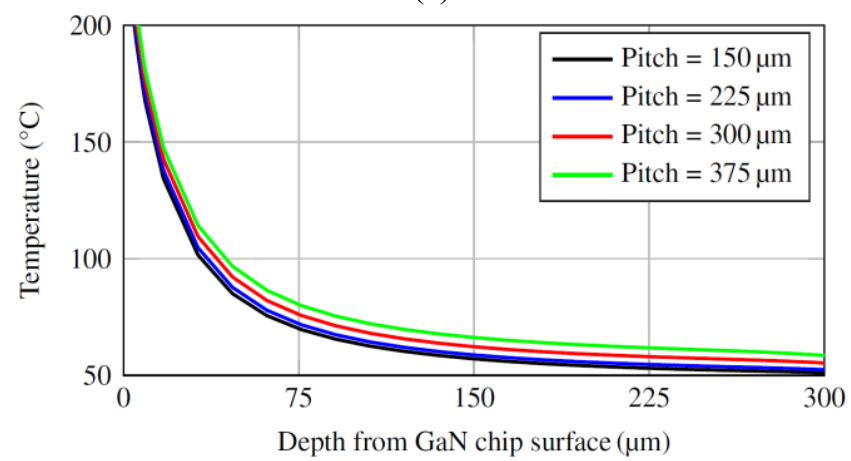

(b)

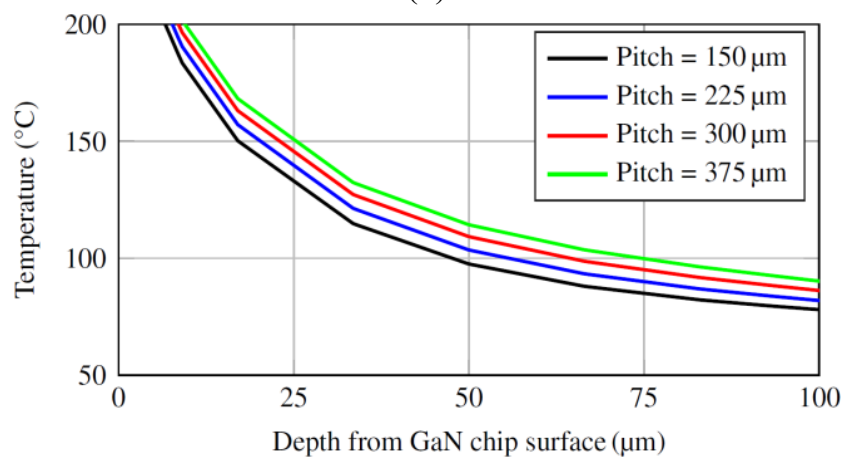

(c)

Figure 6. Vertical temperature profiles at the centre of the $\mathrm{GaN} / \mathrm{SiC}$ chip with different bump pitch values when the $\mathrm{SiC}$ carrier thickness is (a) $500 \mu \mathrm{m}$, (b) $300 \mu \mathrm{m}$, and (c) $100 \mu \mathrm{m}$. The red line in the inset shows the direction of the temperature profile.

With all the studied SiC thicknesses, the sparsest bump pitch results in a slightly anomalous behaviour at the $\mathrm{GaN} / \mathrm{SiC}$ interface. This could be resulting from a locally reduced heat transfer caused by increased distance to the adjacent bump.

To illustrate the vertical temperature drop at different locations on the chip, Figure 8 represents the vertical temperature difference $\left(\Delta T_{\text {vert }}=T_{\text {GaN,top }}-T_{\mathrm{SiC}, \text { bottom }}\right)$ as a function of lateral distance from the heat source. The curves show that the vertical temperature difference reduces when moving away from the source, as expected, and that the profile is smoother with a denser bump grid.

When reducing the number of the bumps, their individual locations gradually become visible in the temperature profile 
as local dips, whose positions correlate well with those of the bumps. With all the investigated substrate thickness and pitch values, the vertical temperature difference is within $2{ }^{\circ} \mathrm{C}$ beyond $0.5 \mathrm{~mm}$ from the chip centre.

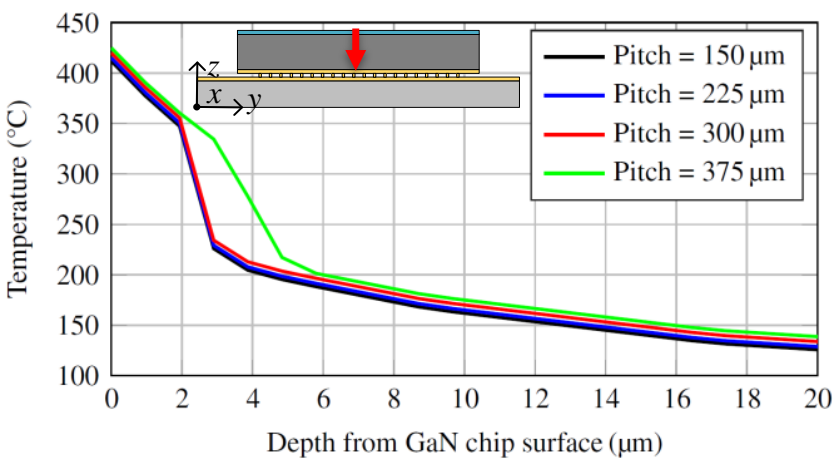

(a)

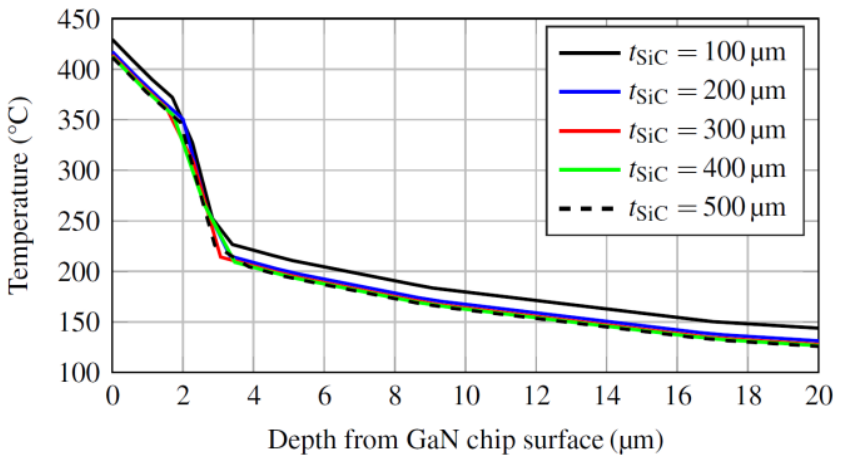

(b)

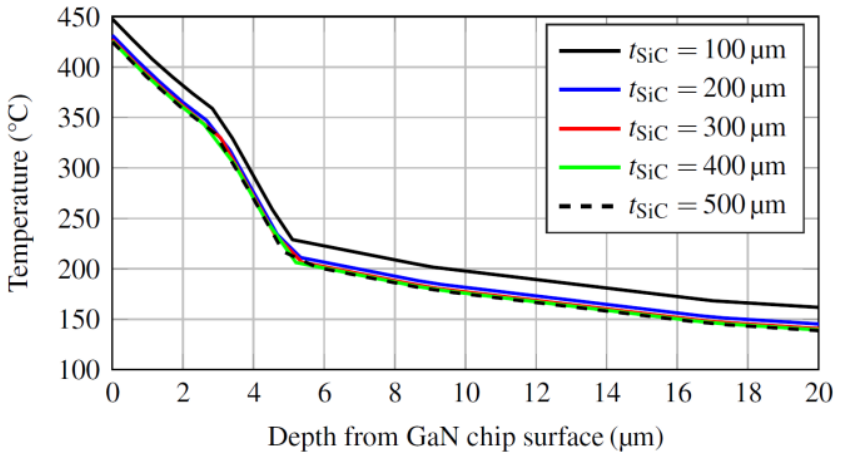

(c)

Figure 7. Close-up of the vertical temperature drop in the vicinity of the GaN layer when (a) $t_{S i C}=500 \mu \mathrm{m}$, (b) pitch $=$ $150 \mu \mathrm{m}$, and (c) pitch $=375 \mu \mathrm{m}$. The red line in the inset shows the direction of the temperature profile.

\section{Discussion}

The observed lateral and vertical thermal effects raise a question of how the thermal characteristics of the studied assembly approach would change if the bump array outside the 0.5 -mm radius were sparsened further. From a practical point of view, this is an important aspect as the currently used "ideal", fully populated bump arrays take up a considerable part of the overall chip area and increase the manufacturing costs for commercial implementations.

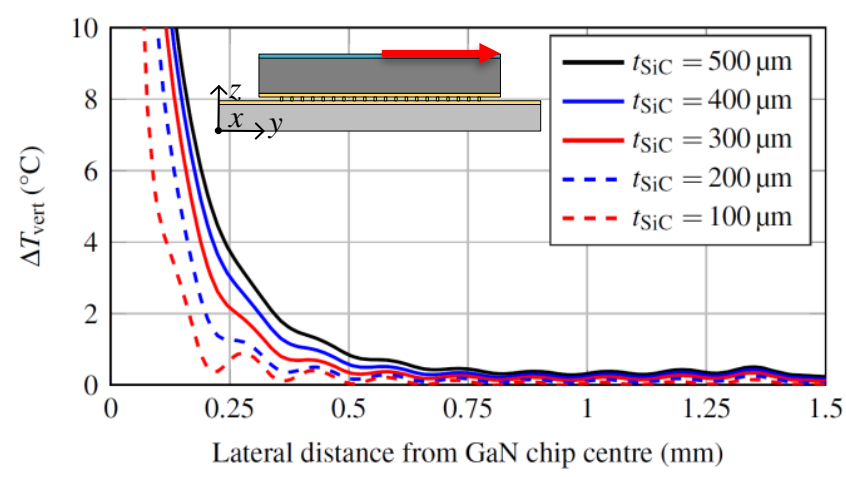

(a)

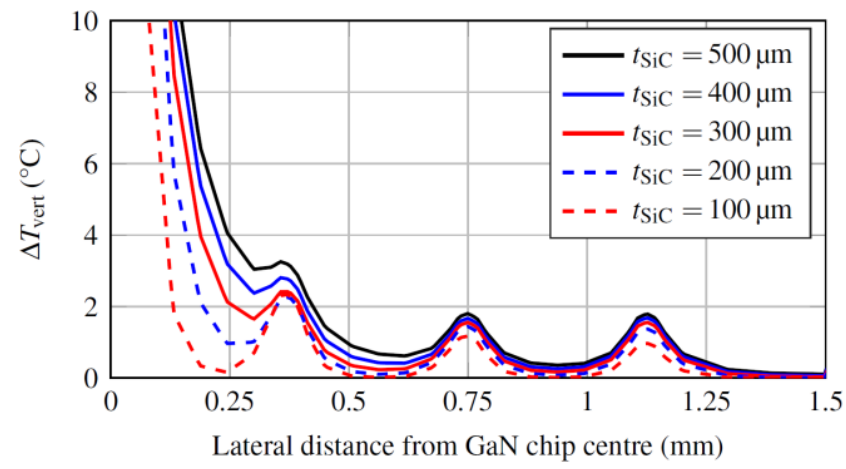

(b)

Figure 8. Vertical temperature difference $\left(\Delta T_{\mathrm{vert}}=T_{\mathrm{GaN}, \mathrm{top}}-\right.$ $\left.T_{\mathrm{SiC}, \text { bottom }}\right)$ as a function of $\mathrm{SiC}$ carrier thickness when the bump pitch is (a) $150 \mu \mathrm{m}$ and (b) $375 \mu \mathrm{m}$. The red line in the inset shows the direction of the temperature profile.

In the present study, the array is only utilised for vertical heat transfer and, to some extent, structural and thermomechanical rigidity (which are outside the scope of this work). A more practical structure would require transferring both heat and RF signals in the vertical direction, meaning that a careful consideration is needed to suitably distribute the available area to incorporate all the required functionalities.

Additionally, the heterogeneous nature of the materials (e.g., different thermal expansion coefficients) used in the assembly can be a problem for the reliability. The structure experiences repetitive heating and cooling cycles during its operation, which causes thermomechanical stress. These aspects should be investigated in a separate study.

It should be noted that some of the assumptions made in the present study, e.g., on the dissipated power and dimensions of the heat source, represent something of a thermal worst-case scenario as the high $P_{\text {diss }}$ is constantly on (steady-state result). In practice, the time-dependent properties of the used signals (power level, pulse duration, duty cycle etc.) can alleviate some of the worst problems, but the resulting possible transient thermal challenges also need to be properly considered. 


\section{Conclusion}

This work has investigated how the heat spreading characteristics of a stacked heterogeneously integrated $\mathrm{GaN} / \mathrm{SiC}$-on-Si assembly are affected by the thickness of the $\mathrm{SiC}$ carrier and the bump pitch. The results show that as the $\mathrm{SiC}$ carrier is thinned down to $100 \mu \mathrm{m}$, its bottom surface heats up more in the vicinity of a highly localised heat source. With the current bump arrays, none of the studied pitches provides major thermal bottlenecks, but the used arrays can be too ideal for practical purposes.

The outcome of this study provides useful insights for designing compact, integrated, and high-power devices whose operation will not be restricted by thermal constraints. Studies on additional parameters and quantities are still needed for obtaining a more detailed understanding of the investigated lateral and vertical heat transfer problem. Future work also includes implementation of test structures for experimental studies on the thermal properties of the proposed assembly and integration approach.

\section{Acknowledgements}

This work was supported by the strategic innovation programme "Smarter Electronic Systems", a joint effort by the Swedish Government Agency for Innovation Systems (VINNOVA), Formas, and the Swedish Energy Agency, through decision number 2017-01898. The authors would like to thank Mr. P. Ingelhag and Dr. P. Melin from Ericsson $\mathrm{AB}$, Sweden, for providing suitable bump dimensions.

\section{Literature}

[1] J. G. Andrews et al., "What will 5G be?" IEEE J. Sel. Areas Commun., vol. 32, no. 6, pp. 1065-1082, Jun. 2014.

[2] E. Hossain and M. Hasan, "5G cellular: Key enabling technologies and research challenges," IEEE Instrum. Meas. Mag., vol. 18, no. 3, pp. 11-21, Jun. 2015.

[3] K. K. Samanta, "Pushing the envelope for heterogeneity: Multi-layer and 3-D heterogeneous integration for next generation millimeter- and submillimeter-wave circuits and systems," IEEE Microw. Mag., vol. 18, no. 2, pp. 28-43, Mar. 2017.

[4] R. Tummala, N. Nedumthakady, S. Ravichandran, B. DeProspo, and V. Sundaram, "Heterogeneous and homogeneous package integration technologies at device and system levels," Proc. 2018 Pan Pacific Microelectronics Symp. (Pan Pacific), Big Island, HI, USA, Feb. 2018, 5 p.

[5] J. Zhou, J. Yang, and Y. Shen, "3D heterogeneous integration technology using hot via MMIC and silicon interposer with millimeter wave application," Proc. IEEE MTT-S Int. Microwave Symp. (IMS), Honolulu, HI, USA, Jun. 2017, pp. 499-502.

[6] Y. Zhang, Y. Zhang, T. Sarvey, C. Zhang, M. Zia, and M. Bakir, "Thermal isolation using air gap and mechanically flexible interconnects for heterogeneous
3-D ICs," IEEE Trans. Compon. Packag. Manuf. Technol., vol. 6, no. 1, pp. 31-39, Jan. 2016.

[7] J. H. Lau et al., "Fan-out wafer-level packaging for heterogeneous integration," IEEE Trans. Compon. Packag. Manuf. Technol., vol. 8, no. 9, pp. 1544-1560, Sep. 2018.

[8] K. Rasilainen, K. Buisman, K. Andersson, and C. Fager, "Multi-physical simulations and modelling of an integrated GaN-on-Si module concept for millimetrewave communications," Proc. 2020 IEEE 70 Electronic Components and Technology Conf. (ECTC), Lake Buena Vista, FL, USA, Jun. 2020, pp. 1369-1375.

[9] C. Bailey and S. Stoyanov, "Co-simulation and modelling for heterogeneous integration of high-tech electronic systems," Proc. 2017 40 ${ }^{\text {th }}$ Int. Spring Seminar Electronics Technology (ISSE), Sofia, Bulgaria, May 2017, 5 p.

[10] J.-M. Jin and S. Yan, "Multiphysics modeling in electromagnetics: Technical challenges and potential solutions," IEEE Antennas Propag. Mag., vol. 61, no. 2, pp. 14-26, Apr. 2019.

[11] T. Nowak, S. Merbold, C. Egbers, and R. Schacht, "Numerical study with experimental validation of thermal coupling phenomena with flip-chip assembled test dies on PCB," Proc. $24^{\text {th }}$ Int. Workshop on Thermal Investigations of ICs and Systems (THERMINIC), Stockholm, Sweden, Sep. 2018, 5 p.

[12] A. El Helou, M. J. Tadjer, K. D. Hobart, and P. E. Raad, "Full 3D thermal simulation of GaN HEMT using ultrafast self-adaptive computations driven by experimentally determined thermal maps," Proc. $24^{\text {th }}$ Int. Workshop on Thermal Investigations of ICs and Systems (THERMINIC), Stockholm, Sweden, Sep. 2018, 3 p.

[13] Y. Zhang, T. E. Sarvey, and M. S. Bakir, "Thermal evaluation of 2.5-D integration using bridge-chip technology: Challenges and opportunities," IEEE Trans. Compon. Packag. Manuf. Technol., vol. 7, no. 7, pp. 1101-1110, Jul. 2017.

[14] Y. Zhang, Y. Zhang, and M. S. Bakir, "Thermal design and constraints for heterogeneous integrated chip stacks and isolation technology using air gap and thermal bridge," IEEE Trans. Compon. Packag. Manuf. Technol., vol. 4, no. 12, pp. 1914-1924, Dec. 2014.

[15] K. Rasilainen, P. Ingelhag, P. Melin, T. M. J. Nilsson, M. Thorsell, and C. Fager, "Thermal characteristics of vertically-integrated GaN/SiC-on-Si assemblies: A comparative study," Proc. 2019 IEEE 69 ${ }^{\text {th }}$ Electronic Components and Technology Conf. (ECTC), Las Vegas, NV, USA, May 2019, pp. 1405-1412.

[16] J. Bremer et al., "Analysis of lateral thermal coupling for GaN MMIC technologies," IEEE Trans. Microw. Theory Techn., vol. 66, no. 10, pp. 4430-4438, Oct. 2018.

[17] M. Garven and J. P. Calame, "Simulation and optimization of gate temperatures in GaN-on-SiC monolithic microwave integrated circuits," IEEE Trans. Compon. Packag. Technol., vol. 32, no. 1, pp. 63-72, Mar. 2009. 